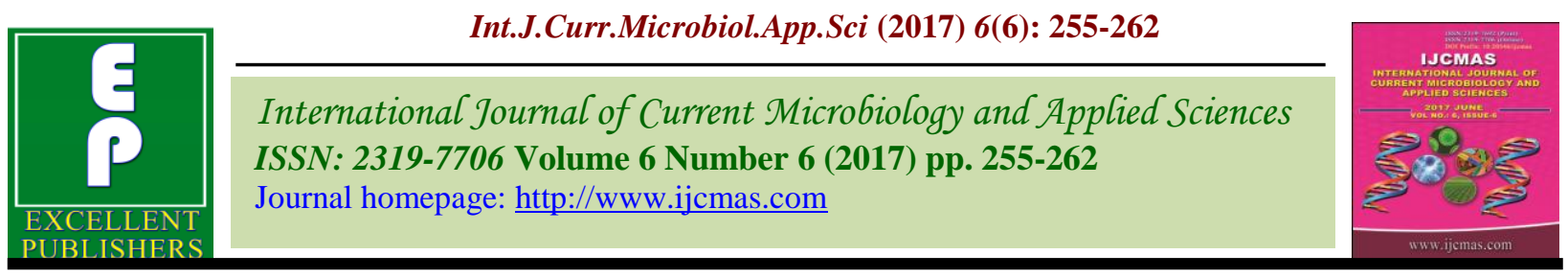

Original Research Article

https://doi.org/10.20546/ijcmas.2017.606.031

\title{
Evaluation of Banana Genotypes under Northern Dry Zone of Karnataka for Yield and Returns
}

\author{
B.S. Sagar*, B. Raju and B.R. Sahithya \\ Department of Fruit science, College of Horticulture Bagalkot, University of Horticultural \\ Sciences Bagalkot, 587-104, Karnataka, India \\ *Corresponding author
}

\section{A B S T R A C T}

Keywords

Evaluation,

Genotypes,

Northern dry

zone.

Article Info

Accepted:

04 May 2017

Available Online:

10 June 2017
Banana is the best-known tropical fruit. It is one of the economically important fruit crops grown in Karnataka in both homestead and commercial farms. The present study was undertaken to find the best banana genotype for northern dry zone of Karnataka with respect to yield and the economics. The study was undertaken with twenty three genotypes. Among which, the genotype Hanuman recorded the maximum bunch weight $(38.77 \mathrm{~kg})$ and yield $\left(43.07 \mathrm{t} \mathrm{ha}^{-1}\right)$ with the highest $\mathrm{B}: \mathrm{C}$ ratio of 3.60:1. The genotype mitli performed poor with the minimum bunch weight $(3.46 \mathrm{~kg})$ and the lowest yield $\left(3.84 \mathrm{tha}^{-1}\right)$. The minimum benefit cost ratio was reported in balbisiana genotype (-0.72:1). Among the genotypes evaluated, the genotype hanuman was found the best with respect to yield and economics under Northern dry zone of Karnataka.

\section{Introduction}

Banana (Musa spp) belongs to the family Musaceae. It is the largest produced and consumed amongst all fruit cultivated in India. It is a crop of subsistence being cultivated from pre historic time in India with great socio-economic significance and is grown in all tropical regions. It provides well balanced diet to millions of people around the globe and also contributes to livelihood through crop production, processing (Singh, 2002) and thus plays a key role in the economy of many developing countries. There is no other fruit in the world, which surpasses banana and plantains either in production tonnage or in trade volume in fresh form (FAO, 1985). Banana is delicious fruit crop grown widely all over Karnataka and most important fruit in the country from the stand point of food value and availability throughout the year. In Karnataka banana is the only fruit crop, which is available throughout the year and consumption rate is also higher than any other fruits. Yield evaluation is important to find a high yielding variety at a particular region. Yield estimation trials provide yield estimates for each genotype in each environment. A large number of banana cultivars are grown in North Karnataka. Though, North Karnataka is having congenial condition for commercial 
cultivation of banana, the average yield and benefit obtained is not satisfactory compared to many other regions. This might be due to improper selection of high yielding varieties and lack of systematic management practices or good management practices. Farmers give importance finally towards the yield and benefit. Hence, an investigation was undertaken to identify the high yielding genotype with good benefit cost ratio under northern dry zone of Karnataka.

\section{Materials and Methods}

Study was carriedout to at Sector-70 (Fruit orchard), Karnataka during 2012-2014 with twenty three genotypes viz., Karibale, Kayipallebale, Rajapuri, Red banana, Rasabale, Elakkibale, Kanayibanasi, Mitli, Bargibale, Balbisiana, Pisanglilin, FHIA-3, Lalchakrakeli, Basrai Dwarf, Monthon, Robusta, Kadali, YangaviKM-5, Sakkarebale, Karpuravalli, Poovan, Pisangawak and Hanuman.

Bagalkot is located in Northern Dry Zone (Zone-3) of Karnataka State at 160101 North latitude, 750421 East longitudes and at an altitude of $542.0 \mathrm{~m}$ above the mean sea level. Bagalkot which comes under zone-3 of region-2 among the agro climatic zone of Karnataka has benefited by both South-West and North-East Monsoons. Individual genotype was taken as a treatment which was replicated thrice and three plants were taken for obsrvation in each replication.

The observations on yield parameters of different genotypes were recorded after harvesting the crop. Banana bunches were harvested with a curved knife when fingers were fully developed and devoid of any ridges on its surface and fingers started to change their color from dark green to light green. The weight of bunch was recorded after harvest and expressed in kilogram. The yield was calculated by multiplying the yield per plant with the total number of plants per hectare and expressed in tonnes per hectare. The objective is to work out the benefit cost ratio for different genotypes based on the expenditure incured and market price of the particular genotype.

\section{Results and Discussion}

\section{Yield}

Table 1 shows that the maximum bunch weight per plant $(38.77 \mathrm{~kg})$ was recorded in the genotype Hanuman and the minimum was recorded in Mitli $(3.46 \mathrm{~kg})$. The increase in bunch weight could be the result of an increase in bunch size. Bigger finger size can be a major factor contributing to the bunch weight. The bunch weight was significantly contributed by plant girth, number of leaves per plant, finger length, number of fingers per hand, number of hands per bunch and number of fingers per bunch as accordance with Deshmukh et al., (2004) recorded the maximum bunch weight in acuminate group. Similar findings were obtained by Biswal et al., (2004) and Devi et al., (2011).

The highest estimated yield per hectare was (43.07 $\mathrm{t} \mathrm{ha}^{-1}$ ) recorded in the genotype Hanuman. Whereas, the lowest yield per hectare was $\left(3.84 \mathrm{t} \mathrm{ha}^{-1}\right)$ recorded in genotype the Mitli. Number of fingers per bunch, finger weight and compactness of bunch leads to increase the yield and also improvement in yield was due to its genetical characters. In general Hanuman genotype belongs to the acuminata (AAA) group. Similar findings were obtained by Deshmukh et al., (2004), Medhi (1994) and Gaidashova et al., (2008). 
Table.1 Performance of banana genotypes in respect of yield

\begin{tabular}{|c|c|c|}
\hline \multirow{2}{*}{ Treatments } & \multicolumn{2}{|l|}{ Yield } \\
\hline & Bunch yield/ plant (Kg) & Yield (t/ ha) \\
\hline $\mathrm{T}_{1}-$ Karibale (AAA) & 10.04 & 11.09 \\
\hline $\mathrm{T}_{2}-$ Kayipalle bale $(\mathrm{ABB})$ & 9.42 & 10.46 \\
\hline $\mathrm{T}_{3}-$ Rajapuri (AAB) & 9.39 & 10.43 \\
\hline $\mathrm{T}_{4}-$ Red banana(AAA) & 8.31 & 9.26 \\
\hline $\mathrm{T}_{5}-$ Rasabale (AAB) & 7.37 & 8.19 \\
\hline $\mathrm{T}_{6}-$ Elakkibale $(\mathrm{AB})$ & 11.97 & 13.29 \\
\hline $\mathrm{T}_{7}-$ Kanayibanasi (AAA) & 12.13 & 13.47 \\
\hline $\mathrm{T}_{8}-\operatorname{Mitli}(\mathrm{AB})$ & 3.46 & 3.84 \\
\hline $\mathrm{T}_{9}-$ Bargibale (AAB) & 17.10 & 18.99 \\
\hline $\mathrm{T}_{10}-$ Balbisiana $(\mathrm{BB})$ & 6.90 & 7.66 \\
\hline $\mathrm{T}_{11}-$ Pisanglilin (AA) & 7.36 & 8.17 \\
\hline $\mathrm{T}_{12}-$ FHIA 3 (AABB) & 22.93 & 25.47 \\
\hline $\mathrm{T}_{13}$ - Lalchakrakeli (AAA) & 22.02 & 24.46 \\
\hline $\mathrm{T}_{14}-$ Basrai Dwarf (AAA) & 20.50 & 22.77 \\
\hline $\mathrm{T}_{15}-$ Monthon $(\mathrm{ABB})$ & 17.27 & 19.18 \\
\hline $\mathrm{T}_{16}-$ Robusta (AAA) & 17.89 & 19.88 \\
\hline $\mathrm{T}_{17}-$ Kadali (AA) & 14.42 & 16.02 \\
\hline $\mathrm{T}_{18}-$ Yangavi KM -5 (AAA) & 15.60 & 17.16 \\
\hline $\mathrm{T}_{19}-$ Sakkarebale $(\mathrm{AB})$ & 10.79 & 11.98 \\
\hline $\mathrm{T}_{20}-$ Karpuravalli (AAB) & 13.54 & 15.04 \\
\hline $\mathrm{T}_{21}-$ Poovan (AAB) & 11.54 & 12.81 \\
\hline $\mathrm{T}_{22}-$ Pisangawak $(\mathrm{ABB})$ & 11.29 & 12.53 \\
\hline $\mathrm{T}_{23}-$ Hanuman (AAA) & 38.77 & 43.07 \\
\hline F- test & $* *$ & ** \\
\hline SEm \pm & 1.03 & 1.16 \\
\hline $\mathrm{CD}(0.05)$ & 2.97 & 3.32 \\
\hline
\end{tabular}

* - Significant at $0.05 \% * *$ - Significant at $0.01 \%$ and $0.05 \%$ 
Table.2 Performance of banana genotypes in respect of yield and returns

\begin{tabular}{|c|c|c|c|c|c|}
\hline Treatments & $\begin{array}{l}\text { Yield } \\
\text { (t/ ha) }\end{array}$ & $\begin{array}{l}\text { Total } \\
\text { Cost/ha } \\
(\text { Rs. })\end{array}$ & $\begin{array}{l}\text { Gross } \\
\text { Income/ } \\
\text { ha (Rs.) }\end{array}$ & $\begin{array}{l}\text { Net } \\
\text { Income/ } \\
\text { ha (Rs.) }\end{array}$ & $\begin{array}{l}\text { Benefit } \\
\text { Cost ratio }\end{array}$ \\
\hline $\mathrm{T}_{1}-$ Pisanglilin (AA) & 8.17 & 108626 & 98112 & -10514 & $-0.09: 1$ \\
\hline $\mathrm{T}_{2}$-Kadali (AA) & 16.02 & 112126 & 176220 & 64094 & $0.57: 1$ \\
\hline $\mathrm{T}_{3}-$ Elakkibale $(\mathrm{AB})$ & 13.29 & 111126 & 332466 & 221340 & 1.99:1 \\
\hline $\mathrm{T}_{4}-\operatorname{Mitli}(\mathrm{AB})$ & 3.84 & 108626 & 34596 & -74030 & $-0.68: 1$ \\
\hline $\mathrm{T}_{5^{-}}$Sakkarebale (AB) & 11.98 & 109626 & 131864 & 22238 & $0.20: 1$ \\
\hline $\mathrm{T}_{6}$-Balbisiana $(\mathrm{BB})$ & 7.66 & 112126 & 30660 & -81466 & $-0.72: 1$ \\
\hline $\mathrm{T}_{7}-$ Karibale (AAA) & 11.09 & 109626 & 122694 & 13067 & $0.11: 1$ \\
\hline $\mathrm{T}_{8}-$ Kanayibanasi (AAA) & 13.47 & 111126 & 134760 & 23634 & $0.21: 1$ \\
\hline $\mathrm{T}_{9}-$ Red banana (AAA) & 9.26 & 112126 & 166176 & 54050 & $0.48: 1$ \\
\hline $\mathrm{T}_{10}$-Lalchakrakeli (AAA) & 24.46 & 109626 & 293570 & 183944 & $1.67: 1$ \\
\hline $\mathrm{T}_{11}$-Basrai Dwarf (AAA) & 22.77 & 109626 & 250530 & 140904 & $1.28: 1$ \\
\hline $\mathrm{T}_{12}-$ Robusta (AAA) & 19.88 & 111126 & 178882 & 67756 & $1.05: 1$ \\
\hline $\mathrm{T}_{13}-$ Yangavi KM -5 (AAA) & 17.16 & 111126 & 155984 & 44858 & $0.55: 1$ \\
\hline $\mathrm{T}_{14}-$ Hanuman (AAA) & 43.07 & 112126 & 516881 & 404755 & $3.60: 1$ \\
\hline $\mathrm{T}_{15}-$ Karpuravalli (AAB) & 15.04 & 112126 & 150429 & 38303 & $0.34: 1$ \\
\hline $\mathrm{T}_{16}-$ Poovan $(\mathrm{AAB})$ & 12.81 & 112126 & 153851 & 41725 & $0.03: 1$ \\
\hline $\mathrm{T}_{17}-$ Rasabale (AAB) & 8.19 & 111126 & 90068 & -21058 & $-0.18: 1$ \\
\hline $\mathrm{T}_{18}-$ Rajapuri (AAB) & 10.43 & 109626 & 125184 & 15557 & $0.14: 1$ \\
\hline $\mathrm{T}_{19}-$ Bargibale (AAB) & 18.99 & 109626 & 208979 & 99353 & 0.90:1 \\
\hline $\mathrm{T}_{20}-$ Monthon $(\mathrm{ABB})$ & 19.18 & 109626 & 172682 & 63056 & $0.57: 1$ \\
\hline $\mathrm{T}_{21}-$ Pisangawak $(\mathrm{ABB})$ & 12.53 & 109626 & 137973 & 28347 & $0.25: 1$ \\
\hline $\mathrm{T}_{22}-$ Kayipallebale $(\mathrm{ABB})$ & 10.46 & 109626 & 94190 & -15435 & $-0.14: 1$ \\
\hline $\mathrm{T}_{23}$-FHIA 3 (AABB) & 25.47 & 112126 & 229277 & 117151 & $1.04: 1$ \\
\hline
\end{tabular}


Inputs incurred during the cultivation of different banana genotypes is calculated upto bunch harvesting stage and divided in three seasons

1. Inputs incurred for early season banana genotypes

\begin{tabular}{|c|c|c|}
\hline No. & Materials/works & $\begin{array}{l}\text { Per hectare } \\
(1,100 \text { pl.) }\end{array}$ \\
\hline $\mathbf{I}$ & Inputs & \\
\hline 1. & Ploughing tractor rent and leveling & 3,000 \\
\hline 2. & Suckers at Rs 3 each & 3,333 \\
\hline 3. & Irrigation/water charges & 6,000 \\
\hline II & Nutrition & \\
\hline 1. & FYM-35 tonnes at Rs 1,570 for $1 \mathrm{t}$ & 55,000 \\
\hline 2. & Urea-200 kg/ha (Rs 5.4/kg) & 1,065 \\
\hline 3. & Single Super Phosphate- $120 \mathrm{~kg} / \mathrm{ha}$ (Rs 7.6/kg) & 918.3 \\
\hline 4. & Murate of Potash- $250 \mathrm{~kg} / \mathrm{ha}$ (Rs $23.8 / \mathrm{kg}$ ) & 5,951 \\
\hline III & Interculture operations & \\
\hline 1. & Earthing up, weeding, desuckering & 5,000 \\
\hline 2. & Staking/propping poles & 1,000 \\
\hline 3. & Plant protection & 1,500 \\
\hline IV & Labour charges & \\
\hline 1. & Digging of pits (Rs 500/100 pits) & 5,555 \\
\hline 2. & Filling the pits and planting the suckers (Rs 100/man day) & 2,000 \\
\hline 3. & Fertilizer application 5 labour (Rs 140 each) & 1,800 \\
\hline 4. & Earthing up, weeding, desuckering & 5,000 \\
\hline 5. & Irrigation 1labour & 3,000 \\
\hline 6. & Staking/propping - (2 laoburs/ha) & 1,000 \\
\hline 7. & Plant protection measures - (1 labour $)$ & 1,000 \\
\hline 8. & Harvesting and mattocking - (5 labours/ha) & 1,500 \\
\hline 9. & Watchman (2 months) & 3,000 \\
\hline \multirow[t]{2}{*}{10.} & Misc. expenses & 2,000 \\
\hline & Total Rs. & $1,08,626$ \\
\hline
\end{tabular}


2. Inputs incurred for mid-season banana genotypes

\begin{tabular}{|c|c|c|}
\hline No. & Materials/works & $\begin{array}{l}\text { Per hectare } \\
(1,111 \text { pl. })\end{array}$ \\
\hline I & Inputs & \\
\hline 1. & Ploughing tractor rent and leveling & 3,000 \\
\hline 2. & Suckers at Rs 3 each & 3,333 \\
\hline 3. & Irrigation/water charges & 6,000 \\
\hline II & Nutrition & \\
\hline 1. & FYM-35 tonnes at Rs 1,570 for $1 \mathrm{t}$ & 55,000 \\
\hline 2. & Urea-200 kg/ha (Rs 5.4/kg) & 1,065 \\
\hline \multirow[t]{2}{*}{3.} & Single Super Phosphate- $120 \mathrm{~kg} / \mathrm{ha}$ (Rs 7.6/kg) & 918.3 \\
\hline & Murate of Potash- $250 \mathrm{~kg} / \mathrm{ha}$ (Rs $23.8 / \mathrm{kg}$ ) & 5,951 \\
\hline III & Interculture operations & \\
\hline 1. & Earthing up, weeding, desuckering & 5,000 \\
\hline 2. & Staking/propping poles & 1,000 \\
\hline 3. & Plant protection & 1,500 \\
\hline IV & Labour charges & \\
\hline 1. & Digging of pits (Rs 500/100 pits) & 5,555 \\
\hline 2. & Filling the pits and planting the suckers (Rs 100/man day) & 2,000 \\
\hline 3. & Fertilizer application 5labour (Rs 140 each) & 1,800 \\
\hline 4. & Earthing up, weeding, desuckering & 5,000 \\
\hline 5. & Irrigation 1labour & 3,000 \\
\hline 6. & Staking/propping - (2 laoburs/ha) & 1,000 \\
\hline 7. & Plant protection measures - (1 labour $)$ & 1,000 \\
\hline 8. & Harvesting and mattocking - (5 labours/ha) & 1,500 \\
\hline 9. & Watchman (3 months) & 4,000 \\
\hline \multirow[t]{2}{*}{10.} & Misc. expenses & 2,000 \\
\hline & Total Rs & $1,09,626$ \\
\hline
\end{tabular}


3. Inputs incurred for late season banana genotypes

\begin{tabular}{|l|l|l|}
\hline No. & Materials/works & $\begin{array}{l}\text { Per hectare } \\
\mathbf{( 1 , 1 1 1} \text { pl.) }\end{array}$ \\
\hline I & Inputs & \\
\hline 1. & Ploughing tractor rent and leveling & 3,000 \\
2. & Suckers at Rs 3 each & 3,333 \\
3. & Irrigation/water charges & 6,500 \\
\hline II & Nutrition & \\
\hline 1. & FYM-35 tonnes at Rs 1,570 for one tone & 55,000 \\
2. & Urea-200 kg/ha (Rs 5.4/kg) & 1,065 \\
3. & Single Super Phosphate- 120 kg /ha (Rs 7.6/kg) & 918.3 \\
4. & Murate of Potash- 250 kg/ha (Rs 23.8/kg) & 5,951 \\
\hline III & Interculture operations & \\
\hline 1. & Earthing up, weeding, desuckering & 5,000 \\
2. & Staking/propping poles & 1,000 \\
3. & Plant protection & 1,500 \\
\hline IV & Labour charges & \\
\hline 1. & Digging of pits (Rs 500/100 pits) & 5,555 \\
2. & Filling the pits and planting the suckers (Rs 100/man day) & 2,000 \\
3. & Fertilizer application 5labour (Rs 140 each) & 1,800 \\
4. & Earthing up, weeding, desuckering & 5,000 \\
5. & Irrigation 1labour & 3,500 \\
6. & Staking/propping - (2 laoburs/ha) & 1,000 \\
7. & Plant protection measures - (1 labour) & 1,000 \\
8. & Harvesting and mattocking - (5 labours/ha) & 1,500 \\
9. & Watchman (4 months) & 5,000 \\
10. & Misc. expenses & 2,000 \\
\hline & Total Rs & $\mathbf{1 , 1 1 , 6 2 6}$ \\
\hline
\end{tabular}

Table 2 represents the economics of banana genotypes. The maximum benefit cost ratio of 3.60:1 was recorded from the genotype Hanuman followed by Lalchakrakeli (1.67:1), Basrai Dwarf (1.28:1), Robusta (1.05) and FHIA 3 (1.04:1). Though, Elakkibale recorded two times lesser yield than Hanuman and one time less than FHIA-3, due to consumer's preference and higher price in the market, it showed second highest benefit cost ratio. Whereas, the minimum (-0.72:1) benefit cost ratio observed in Balbisiana and Mitli $(-0.68: 1)$. Negative values indicate that genotype like Balbisiana is not preferred by consumers and they have no market in Northern Karnataka compared to dessert type acuminata genotypes. But cooking varieties can't be neglected as they have good market in other states like Tamil Nadu and Kerala.

In conclusion, total cost per hectare (Rs) varied according to the genotypes based on their crop duration. The genotypes with less crop duration consumed less inputs like irrigation water and labour cost and finally less cost per hectare but the total cost was maximum for the genotypes with more crop duration. Among the genotypes evaluated the genotype Hanuman was found the best in terms of bunch weight, total yield per hectare and the maximum benefit cost ratio. Hence, this genotype can be commercialised under Northern dry zone of Karnataka. 


\section{References}

Ara, N., Basher, M. K. and Hossain, M. F.: Growth, yield and quality of banana (Musa sapientum) influenced by different banana varieties/lines and planting time. Trop. Agric. Res. Ext., 14 (2011).

Biswal, M. K., Lenka, P. C. and Dash, D. K.: Evaluation of culinary banana genotypes. Orissa J. Hort., 32: 63-65 (2004).

Deshmukh, S. S., Badgujar, C. D. and Dusane, S. M.: Growth analysis of introduced banana varieties under Jalgaon condition. Agric. Sci. Digest, 23: 233-234 (2004).

Devi, P. S., Thangam, M., Ladaniya, M. S. and Korikanthimath, V. S.: Evaluation of local banana cultivars under coconut shade in Goa. J. Biol. Chem. Research, 28: 63-76 (2011).

FAO: Trade Year Book, Food and Agriculture Organization, Rome, 39: 133-196 (1985).

Gaidashova, S.V., Karemera, F. and Karamura, E. B.: Agronomic performance of introduced banana varieties in lowlands of Rwanda (Uganda). African Crop Sci. J., 16: 9 16 (2008).
Medhi, G.: Performance of some cultivars of banana in Assam. Haryana J. Hort. Sci., 23: 181-185 (1994).

Mustaffa, M.M.: Effect of spacing and nitrogen on growth, fruit and yield of Robusta banana grown under rainfed conditions. South Indian Hort., (3695): 228-231 (1988).

Njuguna, J., Nguthi, F., Wepukhulu, S., Wambugu, F., Gitau, D., Karuoya, M. and Karamura, D.: Introduction and evaluation of improved banana cultivars for agronomic and yield characteristics in Kenya. African Crop Sci. J., 16: 35 40 (2008).

Oliveira, E., De, S. S., Passos, A. R., Donato, S. L. R., Salomao, L. C. C., Pereira, L. V., Rodrigues, M. G. V., Neto, F. P. and Lima, M. B.: Evaluation of banana genotypes in different environments. J. Ciencia Agrotecnologica, 27: 737-748 (2003).

Sarma, A. K. and Roy, A. R.: Fertilizer-cumspacing trial on banana (Musa paradisiaca L.). Ind. J. Agric. Sci., 41:493-496 (1972).

Syamal, M. M. and Mishra, K. A.: Studies on some dessert banana (Musa sapientum L.) cultivars. Ind. J. Hort., 46: 316-318 (1989).

\section{How to cite this article:}

Sagar, B.S., B. Raju and Sahithya, B.R. 2017. Evaluation of Banana Genotypes under Northern Dry Zone of Karnataka for Yield and Returns. Int.J.Curr.Microbiol.App.Sci. 6(6): 255-262. doi: https://doi.org/10.20546/ijcmas.2017.606.031 